\title{
Early Physiotherapy Rehabilitation in Patient with Greater Tuberosity Avulsion Re-Fractures Decreases Kinesiophobia: A Case Report
}

\author{
Mrinmayee Deshmukh ${ }^{1}$, Sanket Mungikar ${ }^{1}$, Kapil Garg $^{2}$, Shripad Joshi $^{3}$ \\ ${ }^{1}$ MPT $2^{\text {nd }}$ year - Masters in Musculoskeletal Physiotherapy, MGM Institute of Physiotherapy, Aurangabad \\ ${ }^{2}$ Associate Professor, Department of Musculoskeletal Physiotherapy, MGM institute of Physiotherapy, \\ Aurangabad \\ ${ }^{3}$ Consultant Orthopedic Surgeon, MGM Medical College, Aurangabad
}

Corresponding Author: Mrinmayee Deshmukh

\begin{abstract}
Background: Patients with hyper flexion/hyper abduction injury to the glen humeral joint are at risk for isolated greater tuberosity fractures. There are typically 2 mechanisms of injury for greater tuberosity fractures: impaction and avulsion injury.

Case Presentation: A 24-year-old male patient sustained a shoulder injury as the result of a fall while driving. Left greater tuberosity avulsion fracture was treated with arthroscopic anchor suture ORIF and GT fixation was done. He was then advised physiotherapy and after one and half month, he again fell from stairs which led to an identical fracture on the same side. ORIF with GT fixation with biotape and biowire was done. Physiotherapy treatment was then resumed after 3-4 days. Active assisted range of motion exercises with core activation, isometrics, and strengthening for shoulder joint with scapular muscle was given. Pre and post physiotherapy treatment outcomes for pain, range of motion, muscle strength, disability and kinesiophobia were measured.

Result: After 2 months of physiotherapy treatment, patient reported decrease in pain, TSK score, SPADI score and improvement in shoulder ranges with muscle strength.

Conclusion: Early physiotherapy after surgery for greater tuberosity avulsion fracture decreased pain and kinesiophobia with improvement of range, muscle strength and functional abilities of shoulder joint.
\end{abstract}

Keywords: Greater tuberosity avulsion fracture, Physiotherapy, kinesiophobia

\section{INTRODUCTION}

In all fractures, proximal humerus fractures account for $4 \%$ to $5 \%$ and isolated greater tuberosity fractures reported as $12 \%$ to $19 \%$ of proximal humerus fractures. ${ }^{1}$ Patients with hyper flexion/hyper abduction injury to the glenohumeral joint are more prone for isolated greater tuberosity fractures. Injury to greater tuberosity fractures might be occurred by two mechanisms: impaction and avulsion injury. ${ }^{2}$

Surgical open reduction internal fixation is the treatment option of choice for greater tuberosity avulsion fractures. The good results observed when an early mobilization and immediate home based self-physiotherapy exercise program are started post surgery. Hence fastens the recovery and decreases dependency as early physical therapy improves shoulder mobility and reduces postoperative pain, disability and kinesiophobia.

Here, we reported case of young male who sustained injury twice to his left shoulder. The purpose of the case study was to describe the management of a male 
patient with greater tuberosity avulsion fracture following surgery.

\section{CASE REPORT}

\section{History:-}

Patient was 24 year old male. He sustained a shoulder injury of left side as the result of a fall on outstretched arm while driving. Pain was started immediately after fall. He had swelling around the shoulder; tenderness was present on the superolateral aspect of the shoulder. On radiological examination of patient, there was an avulsion fracture of the greater tuberosity. Patient was informed about surgery and he agreed with early intervention. Surgery was performed by an orthopaedic surgeon. Arthroscopic anchor suture and open reduction internal fixation for greater tuberosity was done. He was immobilized for $1 \& 1 / 2$ month. After immobilization, he again fell from stairs which led to refracture. ORIF with GT fixation by biotape and biowire was done. Physiotherapy treatment was then started after 3-4 days.

\section{Clinical Findings}

The patient presented with pain around incisional site.

Pain intensity: - 8 on Visual analogue scale (VAS)

Range of motion (ROM) of the shoulder was as follows:-

\begin{tabular}{|l|l|l|}
\hline & Active ROM & Passive ROM \\
\hline Flexion & $0^{\circ}-10^{\circ}$ & $0^{\circ}-85^{\circ}$ \\
\hline Extension & $0^{\circ}-26^{\circ}$ & $0^{\circ}-45^{\circ}$ \\
\hline Abduction & $0^{\circ}-34^{\circ}$ & $0^{\circ}-78^{\circ}$ \\
\hline Internal rotation & Not indicated & \\
\hline External rotation & Not indicated & - \\
\hline
\end{tabular}

Manual muscle tests (MMT) for

1. Flexors:- $2+$

2. Extensors :-2+

3. Abductors:- $2+$

The shoulder pain and disability index (SPADI) score was $80 \%$.

The Tampa scale of kinesiophobia (TSK) score was 39.

\section{Diagnostic assessment}

Radiographic investigations determined left greater tuberosity avulsion fracture.

\section{Therapeutic intervention}

The patient was seen 3 times a week over 8 weeks.

\section{Initial treatment:-}

- Patient education

- TENS for pain reduction. Two electrodes were placed around the incision approximately two inches from the proximal and distal ends of the surgical incision prior to each exercise session. A frequency of 150 pulse per second with pulse duration of $150 \mu \mathrm{s}$ was used and patient was instructed to have the highest tolerable intensity for 20 minutes.

- Active assisted range of motion exercise-

1. The finger ladder exercises for shoulder flexion and abduction was performed 10-15 repetitions of each.

2. Wand exercises were performed for shoulder flexion, abduction and extension in pain free range 10-15 repetitions of each. Internal and external rotation for shoulder joint was initiated after removal of stitches.

- The pendulum or Codman's exercise was performed with the trunk flexed and the right arm on table or couch to prevent falls, the left injured upper extremity hangs perpendicular to the floor due to gravity. Gently, the right limb is moved loosely in circles, from side to side, and forward-backward

- Pain-free submaximal isometrics for shoulder were given

- Core activation exercise- Draw in abdomen exercise during active assisted shoulder flexion, abduction and extension

Patient was encouraged to perform the exercise program at least 2 times each day, with 10 to 15 repetitions for each exercise. 
Mrinmayee Deshmukh et.al. Early physiotherapy rehabilitation in patient with greater tuberosity avulsion refractures decreases kinesiophobia: a case report.

Progression was done after 3 weeks with no pain:-

- Active shoulder exercises- Shoulder flexion in supine, abduction in side lying and extension in prone was started. Internal and external rotation for shoulder joint was done in sitting. Horizontal abduction and adduction was initiated.

- Multiple angle isometrics- Isometrics to shoulder flexion abduction and extension at 3 angle was started in available range of motion.

- Scapular strengthening exercises- T to $\mathrm{Y}$ to $\mathrm{W}$ exercise

- Closed kinematic chain exercises (CC)wall pushup exercise

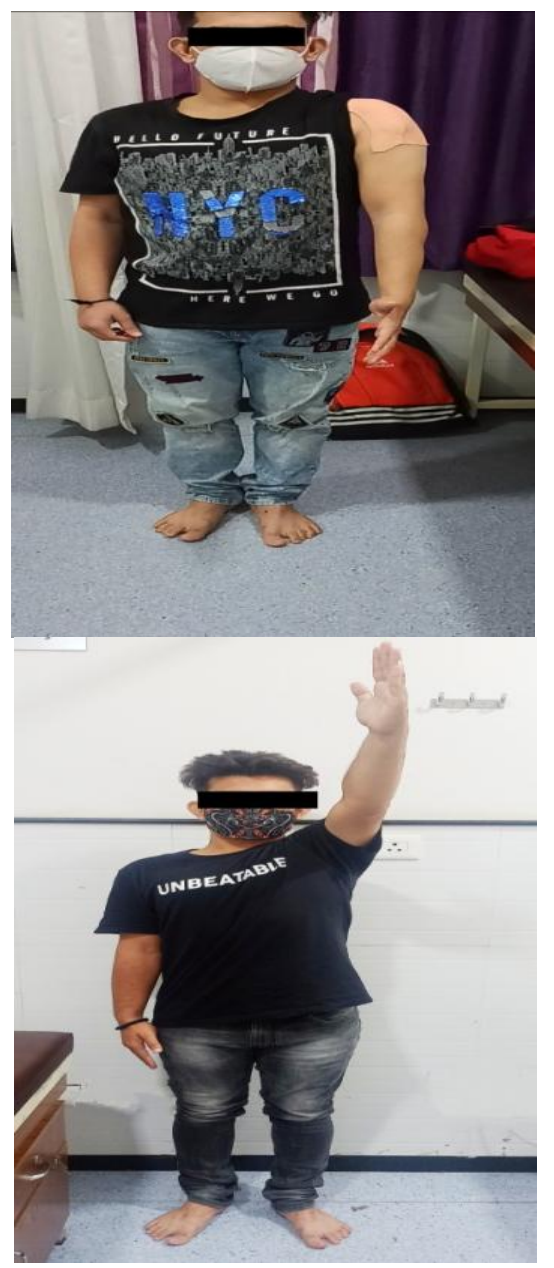

Pre and post treatment shoulder range of motion.

DISCUSSION

Reports

on physiotherapy management of greater tuberosity avulsion fracture are discussed.
Strengthening exercises with weightas MMT of shoulder muscles was achieved as grade $3+$, resistance training started with weight cuff according of patient $1 \mathrm{RM}$.

\section{Follow up and outcome}

\begin{tabular}{|l|l|l|}
\hline Outcome & Pre treatment & Post treatment \\
\hline VAS & 8 & 0 \\
\hline ROM of shoulder joint & & \\
\hline Flexion & $0^{\circ}-10^{\circ}$ & $0^{\circ}-132^{\circ}$ \\
\hline Extension & $0^{\circ}-26^{\circ}$ & $0^{\circ}-40^{\circ}$ \\
\hline Abduction & $0^{\circ}-34^{\circ}$ & $0^{\circ}-106^{\circ}$ \\
\hline Internal rotation & $0^{\circ}$ & $0^{\circ}-68^{\circ}$ \\
\hline External rotation & 0 & $0^{\circ}-20^{\circ}$ \\
\hline
\end{tabular}

\begin{tabular}{|l|l|l|}
\hline MMT & & \\
\hline Flexors & $2+$ & 4 \\
\hline Extensors & $2+$ & $3+$ \\
\hline Abductors & $2+$ & $3+$ \\
\hline Internal rotators & - & 3 \\
\hline External rotators & - & 3 \\
\hline TSK & 39 & 20 \\
\hline SPADI & $84 \%$ & $38 \%$ \\
\hline
\end{tabular}
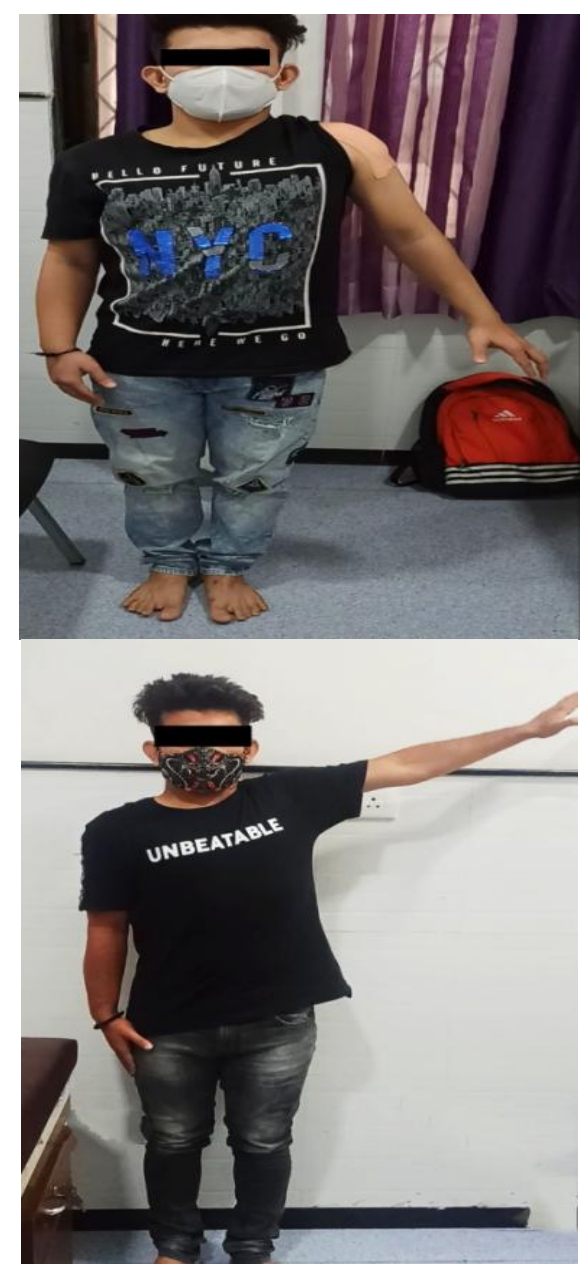

Patient reported no pain on VAS after physiotherapy management. Application of TENS significantly reduced post operative pain by the gate control 
theory of pain. Siddharth A. Mahure et.al ${ }^{3}$, reported significant reductions in post operative pain scores in patients with arthroscopic rotator cuff repair after use of TENS. In study of Jifeng Li, MM et. $\mathrm{al}^{4}$, TENS significantly reduced pain after total knee arthroplasty. Early shoulder pain-free submaximal isometrics following surgery are given in the acute phase to minimize shoulder muscle atrophy. Early mobility in the protected ranges may promote healing; enhance collagen organization, and helps in decreasing pain.

Reduction in pain and mechanical reduction in edema, and increased extensibility of the shoulder musculotendinous structure improved ROM of shoulder joint. Pendulum and active assisted exercises start recovering the range of shoulder joint and avoiding the development of fibrous scar tissue. In a progressive way, active assisted exercise aids in increase the range of motion. Hector J. Aguado et. $\mathrm{al}^{5}$ reported increase in shoulder joint ranges by performing pendulum and active assisted exercises.

MMT grades improved after treatment. The muscle strength started recovering with active assisted exercise because it can be done from active assisted to active exercise. Hector J. Aguado et. $\mathrm{al}^{5}$ reported improvement in MMT grades with active assisted exercises. Strengthening exercises restores dynamic joint stability. Closed kinetic chain exercise (CC) generated greater activation of shoulder muscles and activates surrounding shoulder musculature and help to restore joint proprioception. Federico Pozzi et.al ${ }^{6}$, supported that Upper extremity closed kinematic chain exercises produced greater activation of shoulder muscle.

Previous studies had found a significant correlation between kinesiophobia and pain intensity. Alejandro Luque-Suarez et. $\mathrm{al}^{7}$, reported association between kinesiophobia and pain intensity and disability with patients of chronic shoulder pain. Reduction in pain may contribute for decrease kinesiophobia and thus decrease in score of SPADI as Avoidance of behaviour based on kinesiophobia can cause physical inactivity lead to increased disability. Patient had kinesiophobia because of the refracture and twice surgery but as pain reduces with improvement of range, muscle strength patient's functional abilities improved. Also, Core musculature becomes active in a feedforward fashion during upper or lower extremity movements. This feed forward mechanism occurs as the body prepares for potential perturbation of spinal stability when extremities begin movement ${ }^{8}$. Draw in abdomen exercise might help to improve functional ability and hence decrease kinesiophobia.

Early physiotherapy for greater tuberosity avulsion refracture surgery decreased pain and kinesiophobia with improvement of range, muscle strength and functional abilities of shoulder joint.

Acknowledgement: None

\section{Conflict of Interest: None}

\section{Source of Funding: None}

\section{REFERENCES}

1. Basim A. N. Fleega, et.al,Arthroscopic Reduction and Transosseous Suture Fixation of Avulsed Displaced Shoulder Greater Tuberosity Fracture, Arthroscopy Techniques, Vol 10, No 3 (March), 2021: pp e821-e830

2. Reg B. Wilcox et.al, Management of a Patient With an Isolated Greater Tuberosity Fracture and Rotator Cuff Tear, Journal of Orthopaedic \& Sports Physical Therapy, 2005;35:521-530

3. Siddharth A. Mahure et.al, Transcutaneous electrical nerve stimulation for postoperative pain relief after arthroscopic rotator cuff repair: a prospective doubleblinded randomized trial, Journal of Shoulder Elbow Surgery, 2017

4. Jifeng Li, MM et. al, Transcutaneous electrical nerve stimulation for postoperative pain control after total knee arthroplasty A meta-analysis of randomized controlled trials, Medicine, 96:37 
Mrinmayee Deshmukh et.al. Early physiotherapy rehabilitation in patient with greater tuberosity avulsion refractures decreases kinesiophobia: a case report.

5. Hector J. Aguado et. al, Does an early mobilization and immediate homebased self-therapy exercise program displace proximal humeral fractures in conservative treatment? Observational study, Journal of Shoulder Elbow Surgery, 2018

6. Federico Pozzi et.al, Electromyography activation of shoulder and trunk muscles is greater during closed chain compared to open chain exercises, Journal of Electromyography and Kinesiology,2019

7. Alejandro Luque-Suarez et. al, Kinesiophobia Is Associated With Pain Intensity and Disability in Chronic Shoulder Pain: A Cross-Sectional Study. $J$
Manipulative Physiol Ther. 2020 Oct;43(8): 791-798. doi: 10.1016/j.jmpt.2019.12.009.

8. Jason Brumitt, Integrating Shoulder and Core Exercises When Rehabilitating Athletes Performing Overhead Activities, Clinical Commentary, 2009

How to cite this article: Deshmukh M, Mungikar S, Garg K et.al. Early physiotherapy rehabilitation in patient with greater tuberosity avulsion re-fractures decreases kinesiophobia: a case report. Int J Health Sci Res. 2021; 11(8): 86-90. DOI: https://doi.org/10.52403/ijhsr. 20210813 